\title{
Osteoarticular tuberculosis: clinical and therapeutic feature
}

\begin{abstract}
Purpose: To review the diagnosis and treatment of osteoarticular tuberculosis (OAT) in 42 patients.

Methods: Records of 23 men and 19 women aged 18 to 83 (mean, 52) years who presented with OAT were reviewed.

Results: Tuberculous spondylodiscitis was diagnosed in 29 patients. An extra-spinal bone tuberculosis was observed in 13 cases touching knees in 5 cases, hip in 5 cases, wrist in one case, sacroiliac joint in one case and greater trochanter in one case. A multifocal osteoarticular tuberculosis was observed in 3 patients. Thirty four patients presented with inflammatory pain. Psoas abscess was noted in 3 cases, paravertebral abscess in 9 cases and multiple abscesses in one case. Radiographs objectified disc space narrowing in 18 cases and erosion of the vertebral endplates in 3 cases. CT was performed in 12 cases and MRI in 22 cases having confirmed bone disease in all cases. The search of koch bacillus in the sputum was positive in 4 cases, in the urine in 2 cases and in joint fluid in 11 cases. An histological confirmation was obtained in 11 cases. The tuberculous origin was retained in other cases based on clinical and radiological data. Surgical drainage of abscesses was performed in 5 cases. All patients responded to antituberculous therapy (ATT).
\end{abstract}

Conclusion: OAT still common in Tunisia because of the endemicity of tuberculosis. There are still severe forms of the disease. Isolation of Mycobacterium tuberculosis is difficult. The diagnosis is often based on a beam of arguments.

Keywords: Tuberculosis, Osteoarticular, Spondylitis, Radiographs, Osteomyelitis, Rheumatology
Volume 4 Issue 5 - 2016

\section{Zeineb Alaya,' Walid Osman, ${ }^{2}$ Nader Naouar, ${ }^{2}$ Mohamed Laziz Ben Ayèche, ${ }^{2}$ Elyès Bouajina'}

'Department of Rheumatology, Faculty of Medicine of Sousse Farhat Hached Hospital,Tunisia

${ }^{2}$ Department of Orthopedics, Faculty of Medicine of Sousse Sahloul Hospital, Tunisia

Correspondence: Zeineb Alaya, Department of Rheumatology, Farhat Hached Hospital, Faculty of medicine of Sousse, lbn el Jazzar Street, 4000 Sousse,Tunisia Email zeinab_hotmail.fr

Received: February 21, 2016 | Published: March 24, 2016

\section{Introduction}

Osteoarticular tuberculosis (OAT) is still common. ${ }^{1}$ and represents $2-5 \%$ of all tuberculosis cases in Europe and the USA and around 10$15 \%$ of extrapulmonary tuberculosis (EPTB) cases. ${ }^{2,3}$ It rather affects spine and weight-bearing joints. ${ }^{4}$ The objective of our study was to describe the clinical, biological, radiological and therapeutic aspects of OAT.

\section{Materials and methods}

This is a retrospective study of 42 cases of OAT collected in the Department of Rheumatology in Sousse in Tunisia over a period of 17 years [1998-2015].

\section{Results}

Our population consists of 23 men and 19 women. The mean age was 52 years. ${ }^{18-83}$ Comorbidities noted were diabetes in 4 cases and chronic renal failure in 2 cases. Tuberculous contact was found in 4 cases. Alteration of the general state and night sweats were found in 5 cases. Symptoms were dominated by inflammatory pain found in 34 cases. Tuberculous spondylodiscitis was diagnosed in 29 patients. Lumbar spine was affected in 16 cases, thoracic spine in 10 cases ans cervical spine in 3 cases. Extra-spinal bone tuberculosis was observed in 13 cases touching knees in 5 cases, hip in 5 cases, wrist in one case, sacroiliac joint in one case and greater trochanter in one case. Three patients had multifocal OAT. Three patients had Psoas absces. Nine patients had paravertebral abscess and one patient have multiple abscesses. A biological inflammatory syndrome was present in 35 patients. Radiographs were abnormal in 23 cases. They objectified disc space narrowing in 18 cases and erosion of the vertebral endplates in 3 cases. CT was performed in 12 cases and MRI in 22 cases having confirmed bone disease in all cases. The tuberculin test was positive in 7 cases. The search of koch bacillus in the sputum was positive in 4 cases, in the urine in 2 cases and in joint fluid in 11 cases. A histological confirmation showed epithelioid granulomas was obtained in 11 cases. The tuberculous origin was retained in other cases based on clinical and radiological data. All patients were put on antituberculous therapy (ATT) (isoniazid, rifampicin, ethambutol, and pyrazinamide) with total treatment duration of 12 months on average. Surgical drainage of abscesses was performed in 5 cases. The outcome was favorable in all patients under ATT and symptoms regressed after 6 to 8 weeks.

\section{Discussion}

The incidence of OAT has increased in recent years in the underdeveloped countries especially due to the increase of HIV infections. ${ }^{5}$ Because of its low incidence in developed countries, the diagnosis is often delayed for months to years. ${ }^{6}$ OAT tuberculosis predominantly affects large joints (hip and knee). ${ }^{1}$ Tuberculous spondylitis represents approximately $50 \%$ of musculoskeletal TB. ${ }^{2}$ OAT affects the elderly in developed countries and young adults in the under developed countries. A history of pulmonary tuberculosis is rare. Clinical signs are dominated by joint swelling. ${ }^{7}$ with monoarthritis affecting the knee, hip or ankle. ${ }^{6}$ Infectious spondylitis should be suspected in inflammatory back pain. ${ }^{5}$ Tuberculous osteomyelitis and arthritis generally result from reactivation of bacilli lodged in bone during the original mycobacteremia of primary infection. ${ }^{8}$ Early diagnosis and prompt treatment are necessary to prevent destruction 
of joints and skeletal deformity. ${ }^{6}$ The diagnosis may be delayed because of non-specific signs or absence of pain. Imaging plays an important role in the diagnosis of OAT. Standard radiographs are to be realized in the first intention, but can remain normal in early disease. Ultrasound (USG) evaluate soft tissue masses, abscesses, joint effusions but remains not specific in the diagnosis of joint TB. CT scan detects bone erosions, soft tissue masses and sequestrum formation. It allows the bone biopsy guide to provide material for histological and bacteriological examination. ${ }^{5}$ Magnetic resonance imaging (MRI) is the best examination to make the diagnosis at an early stage by showing intra-osseous abscess, soft tissue abscess, tenosynovitis. ${ }^{\text {? }}$

When there is doubt because of an atypical clinical presentation or lack of clinical exposure, histological examination following biopsy of bony lesion, synovium or soft tissue masses is very useful. ${ }^{7,10}$ Diagnosis by biopsy is done in around $46 \%$ of cases. ${ }^{11}$ PCR is a highly sensitive and specific test for the detection of OAT. ${ }^{12,13}$ ELISPOT. ${ }^{14}$ and the IGRA assay. ${ }^{15}$ can also detect OAT.

The treatment is based on antituberculous therapy (ATT). ${ }^{10,16}$ with isoniazid, rifampin, pyrazinamide, and streptomycin or ethambutol. ${ }^{6}$ Treatment duration should be at least 9 months. ${ }^{6}$ Treatment gives good results with low morbidity and mortality. ${ }^{17}$ and approximately $90 \%$ to $95 \%$ of patients heal without sequelae if treated at an early stage. ${ }^{10}$ Surgery is indicated when the patient is unresponsive after 4 to 5 months of ATT, in patients with large abscesses or neurological manifestations or deformities or healing gave a painful joint ankylosis. Surgical operations carried out in these cases are: synovectomy, excisional arthroplasty for the hip or the elbow, arthrodesis for the ankle, the wrist, or the knee or joint replacement if the disease has remained inactive for more than 10 years. ${ }^{5,6,10}$ The results of prolonged medical treatment coupled with surgery gives satisfactory results in most patients. ${ }^{5}$ The few cases reviewed of osteoarticular multidrugresistant tuberculosis have a good outcome with second-line antituberculous drugs combined with surgery. ${ }^{18}$

\section{Conclusion}

OAT still common in Tunisia because of the endemicity of tuberculosis. There are still severe forms of the disease. Isolation of Mycobacterium tuberculosis is difficult. The diagnosis is often based on a beam of arguments.

\section{Acknowledgments}

None.

\section{Conflicts of interest}

None.

\section{References}

1. Vanhoenacker F, Sanghvi D, De Backer A Imaging features of extraaxial musculoskeletal tuberculosis. Indian J Radiol Imaging 2009:19(3):176-186.
2. Houshian S, Poulsen S, Riegels-Nielsen P Bone and joint tuberculosis in Denmark: increase due to imm:igration. Acta Orthop Scand 2000:71(3):312-315.

3. Peto HM, Pratt RH, Harrington TA et al. Epidemiology of extrapulmonary tuberculosis in the United States, 1993-2006. Clin Infect Dis Off Publ Infect Dis Soc Am 2009:49(9):1350-1357.

4. Yoon HJ, Song YG, Park WI et al. Clinical manifestations and diagnosis of extrapulmonary tuberculosis. Yonsei Med J 2004:45(3):453-461.

5. Pigrau-Serrallach C, Rodríguez-Pardo D Bone and joint tuberculosis. Eur Spine J. 2013:22(S4):556-566.

6. Sequeira W, Co H, Block JA Osteoarticular tuberculosis: current diagnosis and treatment. Am J Ther 2000:7(6):393-398.

7. $\mathrm{N} \mathrm{A}$, Ahmad F, Huda $\mathrm{N}$ Osteoarticular tuberculosis-a three years' retrospective study. J Clin Diagn Res.2013:7(10):2189-2192.

8. Gardam M, Lim S Mycobacterial osteomyelitis and arthritis. Infect Dis Clin North Am. 2005:19(4):819-830.

9. Prakash M, Gupta P, Sen RK et al. Magnetic res:onance imaging evaluation of tubercular arthritis of the ankle and foot. Acta Radiol Stockh Swed. 2015:56(10):1236-1241.

10. Tuli SM General principles of osteoarticular tuberculosis. Clin Orthop. 2002:398:11-19.

11. Muangchan C, Nilganuwong S The study of clinical manifestation of osteoarticular tuberculosis in Siriraj Hospital, Thailand. J Med Assoc Thai 92.2009: Suppl 2:S101-S109.

12. Titov AG, Vyshnevskaya EB, Mazurenko SI et al. Use of polymerase chain reaction to diagnose tubercular arthritis from joint tissues and synovial fluid. Arch Pathol Lab Med. 2004:128(2):205-209.

13. García-Elorriaga G, Martínez-Elizondo O, Rey-Pineda G del et al. Clinical, radiological and molecular diagnosis correlation in serum samples from patients with osteoarticular tuberculosis. Asian Pac J Trop Biomed. 2014:4(7):581-585.

14. Wu X, Ma Y, Li D, Yang Y et al. Diagnostic value of ELISPOT technique for osteoarticular tuberculosis. Clin Lab. 2014:60(11):1865-1870.

15. Jia H, Pan L, Qin $\mathrm{S}$ et al. Evaluation of interferon- $\gamma$ release assay in the diagnosis of osteoarticular tuberculosis. Diagn Microbiol Infect Dis. 2013:76(3):309-313.

16. Sharma SK, Mohan A Extrapulmonary tuberculosis. Indian J Med Res. 2004:120(4):316-353.

17. Hodgson SP, Ormerod LP Ten-year experience of bone and joint tuberculosis in Blackburn 1978-1987. J R Coll Surg Edinb. 1990:35(4):259-262.

18. Suárez-García I, Noguerado A Drug treatment of multidrug-resistant osteoarticular tuberculosis: a systematic literature review. Int $J$ Infect Dis IJID Off Publ Int Soc Infect Dis. 2012:16(11):e774-e778 